\title{
INSTABILITY AND NONEXISTENCE OF GLOBAL SOLUTIONS TO NONLINEAR WAVE EQUATIONS OF THE FORM
}

$$
\begin{gathered}
P u_{t t}=-A u+F(u) \\
\text { BY } \\
\text { HOWARD A. LEVINE (1) }
\end{gathered}
$$

\begin{abstract}
For the equation in the title, let $P$ and $A$ be positive semidefinite operators (with $P$ strictly positive) defined on a dense subdomain $D \subseteq H$, a Hilbert space. Let $D$ be equipped with a Hilbert space norm and let the imbedding be continuous.

Let $F: D \rightarrow H$ be a continuously differentiable gradient operator with associated potential function 9 . Assume that $(x, \mathscr{F}(x)) \geq 2(2 \alpha+1) \mathcal{G}(x)$ for all $x \in D$ and some $\alpha>0$.

Let $E(0)=\frac{1}{2}\left[\left(u_{0}, A u_{0}\right)+\left(v_{0}, P v_{0}\right)\right]$ where $u_{0}=u(0), v_{0}=u_{t}(0)$ and $u:[0, T) \rightarrow D$ be a solution to the equation in the title. The following statements hold:

If $G\left(u_{0}\right)>E(0)$, then $\lim _{t \rightarrow T^{-}}(u, P u)=+\infty$ for some $T<\infty$. If $\left(u_{0}, P v_{0}\right)>0,0$ $<E(0)-Q\left(u_{0}\right)<\alpha\left(u_{0}, P v_{0}\right)^{2} / 4(2 \alpha+1)\left(u_{0}, P u_{0}\right)$ and if $u$ exists on $[0, \infty)$, then $(u, P u)$ grows at least exponentially. If $\left(u_{0}, P v_{0}\right)>0$ and $\alpha\left(u_{0}, P v_{0}\right)^{2} / 4(2 \alpha+1)\left(u_{0}, P u_{0}\right) \leq E(0)$ $-\mathcal{G}\left(u_{0}\right)<\frac{1}{2}\left(u_{0}, P v_{0}\right)^{2} /\left(u_{0}, P u_{0}\right)$ and if the solution exists on $[0, \infty)$, then $(u, P u)$ grows at least as fast as $t^{2}$.

A number of examples are given.
\end{abstract}

I. Introduction. A number of authors (Jörgens [7], Keller [9], Sattinger [14] have shown that solutions to the initial value problem or to initial-boundary value problems for classical nonlinear wave equations in one, two or three dimensions are not stable in time for arbitrary initial data and arbitrary nonlinearities. Their proofs of these results are based upon a comparison principle together with a Huyghens principle coupled with solving an initial value problem for an associated ordinary differential equation in time. The size of the nonlinearity generally determines the escape time in their proofs.

It is the purpose of this paper to prove similar nonexistence and instability theorems for a wide class of initial and initial-boundary value problems which take the form

$$
P d^{2} u / d t^{2}=-A(t) u+\mathscr{F}(u), \quad t \in[0, T), u(0)=u_{0}, u_{t}(0)=v_{0},
$$

where $u$ is a Hilbert space valued function of $t, A(t)$ is a symmetric linear operator defined and nonnegative for each $t \geq 0, P$ is a strictly positive symmetric operator and $\mathscr{F}$ is a given nonlinearity.

Received by the editors September 1, 1972 and, in revised form, January 8, 1973.

AMS (MOS) subject classifications (1970). Primary 35B05, 35B30, 35L15, 35L60; Secondary 34A15, 34G05, 58F10, 93C20.

(1) This research was supported in part by the Battelle Institute, Advanced Studies Center of Geneva, Switzerland, and in part by the Science Research Council of Great Britain at the University of Dundee, Dundee, Scotland, as part of the activities of the North British Symposium on Partial Differential Equations and Their Applications held during the academic year 1971-72. 
Our result says roughly this. Let $u$ be a twice strongly continuously differentiable function satisfying (I-1) on $[0, T)$. Suppose $\mathscr{F}$ has a symmetric Fréchet derivative $\mathscr{F}_{x}$ so that the scalar valued function

$$
\mathcal{G}(x) \equiv \int_{0}^{1}(\mathscr{F}(\rho x), x) d \rho
$$

is an appropriate corresponding "potential" for $\mathscr{F}$. Suppose further that there is a constant $\alpha>0$ such that $(x, \mathscr{F}(x)) \geq 2(2 \alpha+1) \mathcal{S}(x)$ for all $x$ in the appropriate domain. Then, whenever

$$
\mathcal{G}\left(u_{0}\right)>\frac{1}{2}\left[\left(u_{0}, A(0) u_{0}\right)+\left(v_{0}, P v_{0}\right)\right] \equiv E(0),
$$

the interval of existence of $u$ is bounded and, for some $T<\infty$,

$$
\lim _{t \rightarrow T^{-}}(u(t), P u(t))=+\infty .
$$

That is to say, if the initial potential energy of the nonlinearity is larger than the total initial energy of the linear problem, then (I-1) cannot have global solutions. If $\mathcal{G}\left(u_{0}\right)<E(0)$ then the stability and global existence questions are open. Theorems III and IV provide a partial answer, however.

In addition to providing a more unified approach to the "negative" theorems of [7], [9] and [14], this approach is of interest because:

(1) The special properties of the classical wave equation (finite speed of propagation, Huyghen's principles) are not invoked. In fact there may not be such principles for general (I-1).

(2) It is not necessary to obtain a first integral of any "space variable" independent version of the wave equation. (This may not be possible as we shall see in one of the examples.)

(3) The results are applicable to higher order equations, systems of equations, equations for which "densities" may have zero lines and surfaces and to various kinds of initial and initial-boundary value problems for which $P$ can be unbounded. (See the examples.)

(4) The nonlinearity can be very "mild". In addition to "polynomials" in the solution, it is even possible to have nonlinear integral operators as nonlinearities. (See Example II.)

(5) The results have a pleasant geometric interpretation (Theorems II and V). Strauss [15] points out that "There are a few examples of solutions which "blow up', that is, which do not exist in the large. In general, however, there is a definite lack of counterexamples which can be used as guides to the theory." It is hoped that this work can provide some such guides as it shows how one may obtain a wide class of examples for which one has unstable solutions.

In [14], Sattinger remarks that large (space) domains seem to be more unstable than small space domains. One of our side results will make more precise his heuristic arguments to that effect (Example III). 
In the next section we state and prove some abstract results about (I-1) and in §III we give some examples, commenting on the literature as we go. In §IV we briefly examine the damped equation

$$
d^{2} u / d t^{2}+a d u / d t=-A u+\mathscr{F}(u)
$$

where $a$ is a constant $(>0)$.

Physically, of course, all our results say the same thing. If you start with "large enough" displacements, and the "energy" is not a definite functional, then your solutions (transverse vibrations, displacement vectors, etc.) cannot exist for all time. The point is that they give sufficient conditions on $\mathscr{F}, u_{0}$ and $v_{0}$ in order to have unbounded solutions to (I-1) in finite time so that our results tell one where not to look in the data space for choices of initial vectors which yield stable solutions. The question of "where to look" in order to get global existence and stability is to a large extent open when the "energy" is indefinite and $\mathscr{F}$ is "nonlinear". If the energy is positive definite, then in many problems (mostly concerned with the nonlinear wave equation) there is a wide literature on such questions. (See [7], [14] and [15] and the references cited therein.)

II. The abstract theorems. Let $H$ be a Hilbert space, which, for convenience of notation, we take to be real. Let $D \subseteq H$ be a dense linear subspace. Let (, ) denote the scalar product on $\boldsymbol{H}$ and let \|\| denote the corresponding norm. Suppose that for each $t \geq 0$ :

(A-I) $A(t): D \rightarrow H$ is a symmetric linear operator.

(A-II) $(x, A(t) x) \geq 0$ if $x \in D$. (Thus $A(t)$ has a selfadjoint extension but we do not use this fact.)

(A-III) If $v:[0, \infty) \rightarrow H$ is strongly continuously differentiable and if, for all $t \geq 0, v(t)$ and $d v(t) / d t \in D$, then $(v(t), A(t) v(t))$ is continuously differentiable and, for all $t \geq 0$,

$$
Q_{A}(v, v)(t) \equiv(d / d t)(v(t), A(t) v(t))-2(d v(t) / d t, A(t) v(t)) \leq 0 .
$$

Assume that

(P-I) $P$ is a symmetric linear operator, $P: D_{P} \rightarrow H$ and that $D \subseteq D_{P} \subseteq H$.

(P-II) $(x, P x)>0$ for all $x \in D_{P}, x \neq 0$.

Suppose further that $D$ is a Hilbert space under a scalar product $(,)_{D}$. Assume that the injection from $D$ into $H$ is continuous as a mapping of Hilbert spaces. That is, there is a constant $c>0$ such that $\|x\| \leq c\|x\|_{D}$ for all $x \in D$. Then we assume that

(F-I) F: $D \rightarrow H$ is continuously differentiable as a function from $D$ (equipped with \|\|$_{D}$ ) into $H$, that the Fréchet derivative $\mathscr{F}_{x}$ is a symmetric, bounded linear operator on $H$ and that $x \rightarrow \mathscr{F}_{x}$ is a strongly continuous map from $D$ into $\mathcal{E}(H)$.

(F-II) Let $\mathcal{S}(x) \equiv \int_{0}^{1}(\mathscr{F}(\rho x), x) d \rho$ denote the potential associated with $\mathscr{F}$. That is, $B: D \rightarrow R$ is the (unique up to a constant) scalar valued function whose Fréchet derivative $\mathcal{G}_{x}$ can be shown to act as follows: 


$$
\mathcal{G}_{x} y=(\mathscr{F}(x), y)
$$

for all $x, y \in D$. Assume that for some $\alpha>0$,

$$
(x, \mathscr{F}(x)) \geq 2(2 \alpha+1) \mathcal{S}(x)
$$

for all $x \in D$.

The rigorous verification of the action of $\mathcal{G}_{x}$ can be carried out directly from the definition. The details, not being germane to this paper, are omitted.

A formula which will be useful in the sequel is the following, valid for $v:[0, T) \rightarrow D$ with a strongly continuous derivative $v_{t}$ likewise taking values in D:

$$
\mathcal{G}(v(t))-\mathcal{S}(v(0))=\int_{0}^{t}\left(\mathscr{F}(v(\eta)), v_{\eta}(\eta)\right) d \eta
$$

(The strong continuity of $v$ and $v_{t}$ are taken in the sense of the norm on $D$.) This follows directly from the chain rule and the action of $\mathcal{G}_{x}$. The following formal proof is nevertheless instructive. Suppressing the $t$ argument,

$$
\begin{aligned}
\frac{d}{d t} \mathcal{G}(v(t)) & =\int_{0}^{1}\left[\rho\left(\mathscr{F}_{\rho v} v_{t}, v\right)+\left(\mathscr{F}(\rho v), v_{t}\right)\right] d \rho \\
& =\int_{0}^{1}\left[\rho \frac{d}{d \rho}\left(\mathscr{F}(\rho v), v_{t}\right)+\left(\mathscr{F}(\rho v), v_{t}\right)\right] d \rho \\
& =\int_{0}^{1} \frac{d}{d \rho}\left[\rho\left(\mathscr{F}(\rho v), v_{t}\right)\right] d \rho \\
& =\left(\mathscr{F}(v(t)), v_{t}(t)\right),
\end{aligned}
$$

where we have used the symmetry of $\mathscr{F}_{x}$ in the second line.

Definition. We say that $u:[0, T) \rightarrow H$ is a solution to $P u_{u}=-A(t) u+\mathscr{F}(u)$ if, for each $t, u(t)$ and $u_{t}(t)$ belong to $D\left(u_{t}\right.$ being the strong derivative of $u$ in the norm \|\|$_{D}$ on $D$ ), $u_{t t}$ exists and is strongly continuous in the sense of the norm on $H$ and takes values in $D_{P}$, and the differential equation is satisfied in the classical sense.

We have

Theorem I. Consider the initial value problem

$$
\begin{aligned}
P d^{2} u / d t^{2} & =-A(t) u(t)+\mathscr{F}(u(t)), \quad t \in[0, T), \\
u(0) & \left.=u_{0}, u_{t}(0)=v_{0} \cdot{ }^{2}\right)
\end{aligned}
$$

(2) It is not necessary for solutions to (II-1) to be unique. If, however, $(x, P x) \geq \lambda(x, x)$ for all $x \in D$ and some $\lambda>0$ and if $\Phi$ is such that the difference $w$ of two solutions satisfies $\left\|w_{n}+A(t) w\right\|$ $\leq K(t)(w, P w)^{1 / 2}$ where $K(t)$ is a locally bounded function on $[0, T)$ depending upon $u$ and $v$, then $w(0)=w_{t}(0)=0$ implies $w=0$. For details see [11] for example. 
Let $u:[0, T) \rightarrow H$ be a solution to this problem in the above sense. (3) Let $P, \mathcal{F}$ and $A(\cdot)$ satisfy the preceding hypotheses. Then, each of the following statements holds:

(A) If

$$
\beta_{0} \equiv 2\left\{\mathcal{G}\left(u_{0}\right)-\frac{1}{2}\left[\left(u_{0}, A(0) u_{0}\right)+\left(v_{0}, P v_{0}\right)\right]\right\}>0,
$$

then the solution can only exist on a bounded interval $[0, T)$ and

$$
\lim _{t \rightarrow T^{-}}(u(t), P u(t))=+\infty
$$

where

$$
T \leq T_{\beta_{0}} \equiv \alpha^{-1}\left\{\left[\beta_{0}\left(u_{0}, P u_{0}\right)+\left(u_{0}, P v_{0}\right)^{2}\right]^{1 / 2}+\left(u_{0}, P v_{0}\right)\right\}^{-1}\left(u_{0}, P u_{0}\right) .
$$

(B) If

$$
\begin{gathered}
\mathcal{S}\left(u_{0}\right)=\frac{1}{2}\left[\left(u_{0}, A(0) u_{0}\right)+\left(v_{0}, P v_{0}\right)\right], \\
\left(u_{0}, P v_{0}\right) /\left(u_{0}, P u_{0}\right)=\lambda>0,
\end{gathered}
$$

then the solution can only exist on a bounded interval $[0, T)$ and $\lim _{t \rightarrow T^{-}}(u(t), P u(t))$ $=+\infty$ where $T \leq(2 \alpha \lambda)^{-1}$.

It is clear from Theorem I that if $u_{0}$ satisfies

$$
\mathcal{S}\left(u_{0}\right)>\frac{1}{2}\left(u_{0}, A(0) u_{0}\right),
$$

then there exist $v_{0}$ 's such that the corresponding solutions are unstable in finite time.

Corollary I-1. Let $\mathscr{F}(s x)=s^{1+\delta} \mathscr{F}(x)$ for some $\delta>0$ and all $x \in D$. Let $\left(x_{0}, \mathscr{F}\left(x_{0}\right)\right)>0$ for some $x_{0} \in D$. Then there are infinitely many vectors $u_{0}$ such that $\left(\mathrm{d}_{0}\right)$ holds.

Proof of Corollary I-1. Choose $s$ so large that

$$
s^{\delta} \mathcal{G}\left(x_{0}\right)=s^{\delta} \int_{0}^{1}\left(\mathscr{F}\left(\rho x_{0}\right), x_{0}\right) d \rho>\frac{1}{2}\left(x_{0}, A(0) x_{0}\right)
$$

( $s \geq s_{0}$ say). Then for any $u_{0}=s x_{0}$ with $s \geq s_{0}$

$$
\mathcal{S}\left(u_{0}\right)=s^{\delta+2} \mathcal{G}\left(x_{0}\right)>\frac{1}{2}\left(u_{0}, A(0) u_{0}\right) .
$$

In most applications $\delta$ and $\alpha$ are related by $\delta=4 \alpha$.

Proof of Theorem I. Let

$$
F(t)=(u(t), P u(t))+Q^{2}+\beta(t+\tau)^{2}
$$

where $Q, \beta$ and $\tau$ are nonnegative constants which we shall leave undefined for

(3) We shall always assume the existence of a solution to (II-1) near $t=0$. 
the moment. Then, suppressing the $t$ argument on the right and using the symmetry of $P$,

$$
F^{\prime}(t)=2\left(u_{t}, P u\right)+2 \beta(t+\tau) \quad\left(u_{t}=d u / d t\right)
$$

and

$$
F^{\prime \prime}(t)=2\left(u_{t}, P u_{t}\right)+2\left(u, P u_{t}\right)+2 \beta
$$

It follows that

$$
\begin{aligned}
F F^{\prime \prime}-(\alpha+1)\left(F^{\prime}\right)^{2}= & 4(\alpha+1) S^{2}+4(\alpha+1) Q^{2}\left[\left(u_{t}, P u_{t}\right)+\beta\right] \\
& +2 F\left\{\left(u, P u_{t t}\right)-(2 \alpha+1)\left[\left(u_{t}, P u_{t}\right)+\beta\right]\right\}
\end{aligned}
$$

where

$$
S^{2}=\left[(u, P u)+\beta(t+\tau)^{2}\right]\left[\left(u_{t}, P u_{t}\right)+\beta\right]-\left[\left(u, P u_{t}\right)+\beta(u+\tau)\right]^{2} \geq 0 .
$$

Now suppose we could show that $F F^{\prime \prime}-(\alpha+1)\left(F^{\prime}\right)^{2} \geq 0$. Then since $\left(F^{-\alpha}\right)^{\prime \prime}$ $=-\alpha F^{-\alpha-2}\left[F F^{\prime \prime}-(\alpha+1)\left(F^{\prime}\right)^{2}\right]$ whenever $F(t) \neq 0$, it would follow that $F^{-\alpha}$ would be concave. Thus, if $F(0) \neq 0$, we would have, for all $t$ for which $u(t)$ exists, $F^{-\alpha}(t) \leq F^{-\alpha}(0)-\alpha t F^{\prime}(0) F^{-\alpha-1}(0)$, since the graph of a concave function must lie below any tangent line. Hence,

$$
F^{\alpha}(t) \geq F^{\alpha+1}(0)\left[F(0)-\alpha t F^{\prime}(0)\right]^{-1}
$$

and therefore as $t \rightarrow T\left(\leq F(0) / \alpha F^{\prime}(0)\right)$ from below (if $F^{\prime}(0)>0$ ), we see that $F(t) \rightarrow+\infty$. This is the crux of the concavity argument ([10], [12]).

Returning to (II-3), define

$$
\begin{aligned}
H(t) & =\left(u, P u_{t t}\right)-(2 \alpha+1)\left[\left(u_{t}, P u_{t}\right)+\beta\right] \\
& =-(u, A u)-(2 \alpha+1)\left[\left(u_{t}, P u_{t}\right)+\beta\right]+(u, \mathscr{F}(u)) .
\end{aligned}
$$

Thus

$$
\begin{aligned}
H^{\prime}(t) & =-\left[Q_{A}(u, u)+2\left(u_{t}, A u\right)+2(2 \alpha+1)\left(u_{t}, P u_{t}\right)\right]+d(u, \mathscr{F}(u)) / d t \\
& =-Q_{A}(u, u)+4 \alpha\left(u_{t}, A u\right)+d(u, \mathscr{F}(u)) / d t-2(2 \alpha+1)\left(u_{t}, \mathscr{F}(u)\right) .
\end{aligned}
$$

Therefore

$$
\begin{aligned}
H^{\prime}(t)= & -(2 \alpha+1) Q_{A}(u, u)+2 \alpha d(u, A u) / d t \\
& +d(u, \mathscr{F}(u)) / d t-2(2 \alpha+1)\left(u_{t}, \mathscr{F}(u)\right)
\end{aligned}
$$

so that, using (i), (ii), the positive semidefiniteness of $A(t),(*)$ and (**),

$$
\begin{aligned}
H(t)= & H(0)+2 \alpha(u, A u)-2 \alpha\left(u_{0}, A(0) u_{0}\right)-(2 \alpha+1) \int_{0}^{t} Q_{A}(u, u) d \eta \\
& +(u, \mathscr{F}(u))-\left(u_{0}, \mathscr{F}\left(u_{0}\right)\right)-2(2 \alpha+1)\left[\mathcal{G}(u)-\mathcal{G}\left(u_{0}\right)\right] \\
\geq & 2(2 \alpha+1)\left\{\mathcal{G}\left(u_{0}\right)-\frac{1}{2}\left[\left(u_{0}, A(0) u_{0}\right)+\left(v_{0}, P v_{0}\right)+\beta\right]\right\} .
\end{aligned}
$$


Now suppose $\left(\mathrm{d}_{1}\right)$ holds. Then with $Q^{2}=0$ and $\beta=\beta_{0}$ we find that $H(t) \geq 0$ and hence $\left(F^{-\alpha}\right)^{\prime \prime}(t) \leq 0$. Also, $F^{\prime}(0)=2\left(u_{0}, P v_{0}\right)+2 \beta_{0} \tau>0$ if $\tau$ is sufficiently large. Thus, the interval of existence cannot, in this case, exceed $T_{\beta_{0}}$ $\equiv F(0) / \alpha F^{\prime}(0)$ in length. That is

$$
T \leq \frac{\left(u_{0}, P u_{0}\right)+\beta_{0} \tau^{2}}{2 \alpha\left(\left(u_{0}, P v_{0}\right)+\beta_{0} \tau\right)^{2}}=f(\tau) .
$$

One finds that $f(\tau)$ has a minimum on the interval $\left(-\left(u_{0}, P v_{0}\right) / \beta_{0},+\infty\right)$ at

$$
\tau=\beta_{0}^{-1}\left\{-\left(u_{0}, P v_{0}\right)+\left[\left(u_{0}, P v_{0}\right)^{2}+\beta_{0}\left(u_{0}, P u_{0}\right)\right]^{1 / 2}\right\}
$$

and this minimum is $\left(\alpha \beta_{0}\right)^{-1}\left\{-\left(u_{0}, P v_{0}\right)+\left[\left(u_{0}, P v_{0}\right)^{2}+\beta_{0}\left(u_{0}, P u_{0}\right)\right]^{1 / 2}\right\}$ so that

$$
T \leq T_{\beta_{0}}=\alpha^{-1}\left\{\left[\beta_{0}\left(u_{0}, P u_{0}\right)+\left(u_{0}, P v_{0}\right)^{2}\right]^{1 / 2}+\left(u_{0}, P v_{0}\right)\right\}^{-1}\left(u_{0}, P u_{0}\right) .
$$

If $\left(d_{1}^{\prime}\right)$ and $\left(d_{2}^{\prime}\right)$ hold, the proof is easier. Let $Q^{2}=0$ and $\beta=0$ so that $\left[F^{-\alpha}(t)\right]^{\prime \prime} \leq 0$ where $F(t)=(u(t), P u(t))$ and $\lim _{t \rightarrow T^{-}}(u(t), P u(t))=+\infty$ where $T \leq F(0) / \alpha F^{\prime}(0)=\left(u_{0}, P u_{0}\right) / 2 \alpha\left(u_{0}, P v_{0}\right)=1 / 2 \alpha \lambda$.

The next theorem provides a geometric reformulation of Theorem $I$ and Corollary I-2.

Theorem II. Let $\mathscr{F}, P$ and $A(\cdot)$ be as in Theorem $\mathrm{I}$ and $u:[0, T) \rightarrow H$ be a solution to (II-1) in the preceding sense corresponding to $u(0)=u_{0}, u_{t}(0)=v_{0}$. Let $\left(\mathrm{d}_{0}\right)$ hold, i.e.

$$
\mathcal{G}\left(u_{0}\right)>\frac{1}{2}\left(u_{0}, A(0) u_{0}\right)
$$

and let $r\left(u_{0}\right)=\sqrt{ } 2\left[\mathcal{S}\left(u_{0}\right)-\frac{1}{2}\left(u_{0}, A(0) u_{0}\right)\right]^{1 / 2}$. Let $S_{u_{0}}=\left\{v_{0} \in D \mid\left(v_{0}, P v_{0}\right)\right.$ $\left.<r^{2}\left(u_{0}\right)\right\}$, and let, for each $T>0$,

$$
S_{T, \mu_{0}}=\left\{v_{0} \in D \mid\left(v_{0}-u_{0} / \alpha T, P\left(v_{0}-u_{0} / \alpha T\right)\right)<r^{2}\left(u_{0}\right)\right\}
$$

denote spheres of radius $r\left(u_{0}\right)$ and centers 0 and $u_{0} / \alpha T$ in $D$. Let $B_{u_{0}}=\left\{v_{0} \in D \mid\right.$ $\left.\left(v_{0}, P v_{0}\right)=r^{2}\left(u_{0}\right)\right\}$. Then statement (A) of Theorem $I$ is equivalent to

(A') If $v_{0} \in S_{u_{0}}$, then $v_{0} \in S_{u_{0}}-S_{T, u_{0}}$ for some $T>0$ and $\lim _{t \rightarrow T^{-}}(u(t), P u(t))$ $=+\infty$.

Statement (B) is equivalent to:

(B') If $v_{0} \in B_{u_{0}}$ and $0<\cos \theta \equiv\left(u_{0}, P v_{0}\right) /\left(u_{0}, P u_{0}\right)^{1 / 2}\left(v_{0}, P v_{0}\right)^{1 / 2}$, then for some $T>0, v_{0} \in B_{u_{0}}-S_{T, u_{0}}$ and $\lim _{t \rightarrow T^{-}}(u(t), P u(t))=+\infty$.

Proof. Note that $\left(\mathrm{d}_{1}\right)$ is satisfied by $v_{0}$ if and only if $v_{0} \in S_{u_{0}}$ while $\left(\mathrm{d}_{1}^{\prime}\right)$ and $\left(\mathrm{d}_{2}^{\prime}\right)$ hold if and only if $v_{0} \in B_{u_{0}}$ and $\left(v_{0}, P u_{0}\right)>0$. It then follows that the solution with initial data $u_{0}, v_{0}$ has some finite escape time $T$ and $T$ satisfies $T \leq T_{\beta_{0}}$ in the first case and $T \leq 1 / 2 \alpha \lambda$ in the second. However, both of these can easily be seen to be equivalent to the statement that $\left(v_{0}-u_{0} / \alpha T, P\left(v_{0}-u_{0} / \alpha T\right)\right) \geq r^{2}\left(u_{0}\right)$ by using the definition of $T_{\beta_{0}}$ and the definition of $\beta_{0}$. The results follow. 
Remark. Theorem II says that if the solution to (II-1) corresponding to initial data $u_{0}, v_{0}$ has finite escape time $T$ and if $\left(v_{0}, P v_{0}\right) \leq r^{2}\left(u_{0}\right)$ then $v_{0}$ lies in the shaded set depicted in Figure I. Note that as $T$ increases the size of this set decreases.

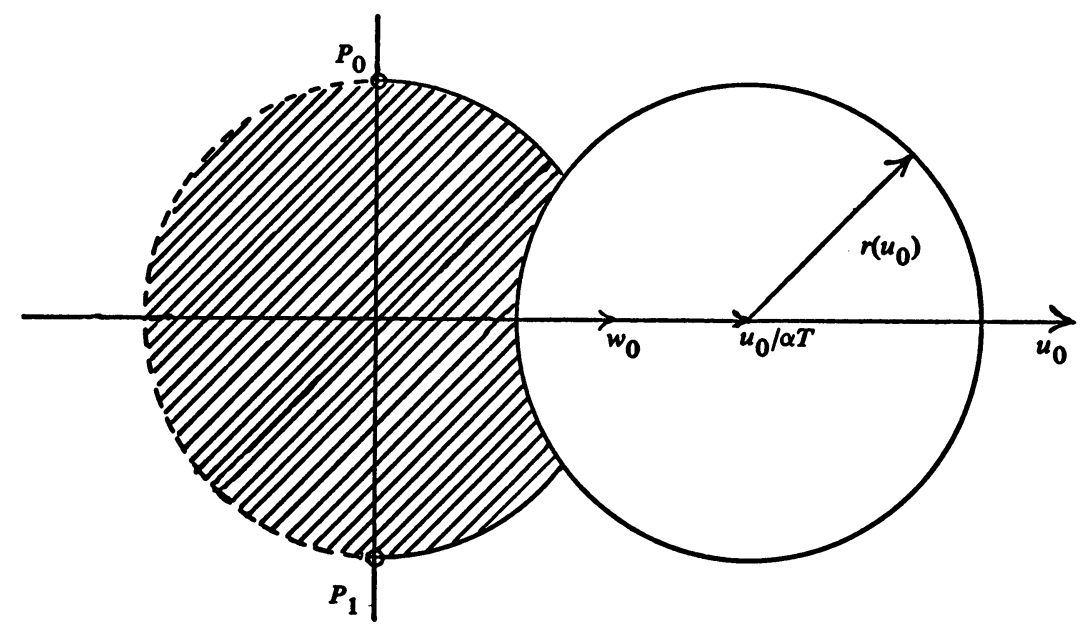

$$
\text { Figure I. }\left(w_{0}=\left(r\left(u_{0}\right) /\left(u_{0}, P u_{0}\right)^{1 / 2}\right) u_{0}\right)
$$

Theorem III. Let $P, \mathcal{F}$ and $A(\cdot)$ be as in Theorem I. Let $u:[0, T) \rightarrow H$ be a solution of (II-1) in the sense of the definition corresponding to $u(0)=u_{0}, u_{t}(0)$ $=v_{0}$. Suppose that

$$
\left(u_{0}, P v_{0}\right) /\left(u_{0}, P u_{0}\right)=\lambda>0
$$

Then if

$$
\mathcal{G}\left(u_{0}\right)<\frac{1}{2}\left[\left(u_{0}, A(0) u_{0}\right)+\left(v_{0}, P v_{0}\right)\right]
$$

and

(d $\left.\mathrm{d}_{4}\right) \quad \frac{1}{2}\left[\left(u_{0}, A(0) u_{0}\right)+\left(v_{0}, P v_{0}\right)\right]-\mathcal{G}\left(u_{0}\right)<\frac{1}{4} \alpha\left(u_{0}, P v_{0}\right)^{2} /(2 \alpha+1)\left(u_{0}, P u_{0}\right)$,

then there is a constant $\gamma>0$ such that

$$
\lim _{t \rightarrow+\infty} \inf e^{-\gamma t}(u(t), P u(t))>0,
$$

whenever this solution exists on $[0, \infty)$.

Proof. Suppose $\left(d_{2}^{\prime}\right),\left(d_{3}\right)$ and $\left(d_{4}\right)$ hold. Let, for arbitrary $\mu>0$,

$$
Q^{2}=4 \mu^{-2}(2 \alpha+1)\left\{\frac{1}{2}\left[\left(u_{0}, A(0) u_{0}\right)+\left(v_{0}, P v_{0}\right)\right]-\mathcal{G}\left(u_{0}\right)\right\}
$$


Then, since $F \geq Q^{2}$, we see that from (II-3) and (II-6) (after putting $\beta=0$ ) that

$$
F F^{\prime \prime}-(\alpha+1)\left(F^{\prime}\right)^{2} \geq-\mu^{2} Q^{2} F \geq-\mu^{2} F^{2} .
$$

For convenience, let $G(t)=F^{-\alpha}(t)\left(G(t)\right.$ is well defined. Observe that $G^{\prime}(0)$ $=-\alpha F^{-\alpha-1}(0) F^{\prime}(0) . G^{\prime}(0)<0$ so that $G^{\prime}(t)<0$ on some interval $[0, \eta)$.

From (II-7) we have

$$
G^{\prime \prime} \leq \alpha \mu^{2} G
$$

and thus, for $t \in[0, \eta)$, we have $G^{\prime}(t)^{2}-G^{\prime}(0)^{2} \geq \alpha \mu^{2}\left(G(t)^{2}-G(0)^{2}\right)$.

Therefore,

$$
\begin{aligned}
\left(G^{\prime}(t)\right. & +\sqrt{ } \alpha \mu G(t))\left(G^{\prime}(t)-\sqrt{ } \alpha \mu G(t)\right) \\
& \geq\left(G^{\prime}(0)+\sqrt{ } \alpha \mu G(0)\right)\left(G^{\prime}(0)-\sqrt{ } \alpha \mu G(0)\right) .
\end{aligned}
$$

Thus, if we can find $\mu>0$ such that

$$
G^{\prime}(0)<-\sqrt{ } \alpha \mu G(0),
$$

then we see from (II-10) and the tacitly assumed smoothness of $u$ that neither factor on the left of (II-9) can change sign and thus, for all $t$ for which the solution exists, $G^{\prime}(t)<-\sqrt{ } \alpha \mu G(t)$. Therefore

$$
G(t) \exp (\sqrt{ } \alpha \mu t) \leq G(0)
$$

or

$$
F(t) \geq F(0) \exp (\mu t / \sqrt{ } \alpha)
$$

and therefore $\lim _{t \rightarrow+\infty} \inf e^{-\gamma t}(u, P u)>0$ with $\gamma=\mu / \sqrt{ } \alpha$. Now one sees from (II-10) that such a $\mu$ exists if and only if there exists $\mu>0$ such that $F^{\prime}(0)>\mu F(0) / \sqrt{ } \alpha$ or such that the quadratic polynomial

$$
\begin{aligned}
P(\mu)= & \mu^{2}\left(u_{0}, P u_{0}\right)-2 \sqrt{ } \alpha\left(u_{0}, P v_{0}\right) \mu \\
& +4(2 \alpha+1)\left\{\frac{1}{2}\left[\left(u_{0}, A(0) u_{0}\right)+\left(v_{0}, P v_{0}\right)\right]-\mathcal{G}\left(u_{0}\right)\right\}
\end{aligned}
$$

is somewhere negative. This will be the case if it has two real roots $\mu_{+}, \mu_{-}$, one of which, $\mu_{+}$, is positive. Then any $\mu \in\left(0, \mu_{+}\right) \cap\left(\mu_{-}, \mu_{+}\right)$will suffice.

Since the coefficient of $\mu$ in the polynomial is negative and since the content of $\left(d_{4}\right)$ is that the discriminant of $P(\mu)$ is positive, the existence of such $\mu$ 's is assured.

Theorem IV. Let $P, \mathcal{F}$ and $A(\cdot)$ satisfy the hypotheses of Theorem I. Let $u:[0, T) \rightarrow D$ be a solution to (II-1) corresponding to $u(0)=u_{0}, u_{t}(0)=v_{0}$ in the sense of the definition. Suppose that condition $\left(\mathrm{d}_{2}^{\prime}\right)$ of Theorem III holds for $u_{0}$ and $v_{0}$ but that $u_{0}$ and $v_{0}$ satisfy 
(ds) $\frac{1}{4} \alpha(2 \alpha+1)^{-1}\left(u_{0}, P v_{0}\right)^{2} /\left(u_{0}, P u_{0}\right)^{2} \leq \frac{1}{2}\left[\left(u_{0}, A(0) u_{0}\right)+\left(v_{0}, P v_{0}\right)\right]-\mathcal{G}\left(u_{0}\right)$,

(d) $\frac{1}{2}\left[\left(u_{0}, A(0) u_{0}\right)+\left(v_{0}, P v_{0}\right)\right]-\mathcal{G}\left(u_{0}\right)<\frac{1}{2}\left(u_{0}, P v_{0}\right)^{2} /\left(u_{0}, P u_{0}\right)$.

Then $\lim _{t \rightarrow+\infty} \inf t^{-2}(u(t), P u(t))>0$ whenever this solution with $u_{0}$ and $v_{0}$ as initial displacement and velocity exists on $[0, \infty)$.

Corollary IV-1. Let $u_{0}, v_{0}$ satisfy $\left(\mathrm{d}_{2}^{\prime}\right),\left(\mathrm{d}_{3}\right)$ and $\left(\mathrm{d}_{6}\right)$. Then $\lim _{t \rightarrow+\infty}(u(t), P u(t))$ $=+\infty$, if $u(\cdot)$ exists on $(0, \infty)$.

The proof of the corollary is an easy consequence of Theorems III and IV.

Proof of Theorem IV. The argument is very similar to that used in Theorem III. Let $Q^{2}=\beta=0$ in (II-2). From (II-3) and (II-6) we obtain

$$
F F^{\prime \prime}-(\alpha+1)\left(F^{\prime}\right)^{2} \geq-2 v^{2}(2 \alpha+1) F
$$

where we have set

$$
\nu^{2}=2\left\{\frac{1}{2}\left[\left(u_{0}, A(0) u_{0}\right)+\left(v_{0}, P v_{0}\right)\right]-\mathcal{S}\left(u_{0}\right)\right\}>0 .
$$

From $\left(\mathrm{d}_{2}^{\prime}\right), F^{\prime}(0)>0$ so that $F^{\prime}(t)>0$ for $t \in[0, \eta)$, say. Multiplying both sides of (II-14) by $-\alpha\left(F^{-\alpha}(t)\right)^{\prime} F^{-(\alpha+2)}(t)^{\prime \prime}$ where $t \in[0, \eta)$ and integrating from 0 to $t$ we obtain

$$
\left[\left(F^{-\alpha}(t)\right)^{\prime}\right]^{2}-4 \alpha^{2} v^{2} F^{-(2 \alpha+1)}(t) \geq\left[\left(F^{-\alpha}(0)\right)^{\prime}\right]^{2}-4 \alpha^{2} v^{2} F^{-(2 \alpha+1)}(0) \text {. }
$$

Since $F(0)=\left(u_{0}, P u_{0}\right)$ and $F^{\prime}(0)=2\left(u_{0}, P v_{0}\right)>0$, the right-hand side of (II-15) will be positive provided that $\left(\mathrm{d}_{6}\right)$ holds as we see after a bit of algebra. If we now factor the left-hand side of (II-15) and argue as was done in passing from (II-9) to (II-11) we find that $\left(F^{-\alpha}\right)^{\prime}<-2 \alpha \nu F^{-(\alpha+1 / 2)}$ for all $t \geq 0$. Hence it follows that

$$
F^{\prime}(t)>2 \nu F^{1 / 2}(t) \text { or } F(t) \geq\left(\nu t+\left(u_{0}, P u_{0}\right)^{1 / 2}\right)^{2}
$$

so that $F$ must grow at least as fast as $t^{2}$.

The next theorem is simply a geometric reformulation of Theorems III and IV.

Theorem V. Let $u_{0}$ satsify $\left(\mathrm{d}_{0}\right)$ and let $S_{u_{0}}$ be as in Theorem II. Let $\cos \theta$ $=\left(u_{0}, P v_{0}\right) /\left(u_{0}, P u_{0}\right)^{1 / 2}\left(v_{0}, P v_{0}\right)^{1 / 2}$ and let

$$
\begin{aligned}
& E_{u_{0}}=\left\{v_{0} \in D \mid\left[1-\left(\frac{1}{2} \alpha /(2 \alpha+1)\right) \cos ^{2} \theta\right]\left(v_{0}, P v_{0}\right)<r^{2}\left(u_{0}\right)\right\}, \\
& H_{+}=\left\{v_{0} \in D \mid\left(u_{0}, P v_{0}\right)>0\right\}, \\
& C_{u_{0}}=\left\{v_{0} \in D \mid\left(v_{0}, P v_{0}\right) \sin ^{2} \theta<r^{2}\left(u_{0}\right)\right\} .
\end{aligned}
$$

Then Theorem III is equivalent to:

(C) If $v_{0} \in H_{+} \cap\left(E_{u_{0}}-S_{u_{0}}\right)$, then any solution to (II-1) corresponding to $u_{0}$ and $v_{0}$ which exists on $[0, \infty)$ must grow at least as fast as $e^{\gamma t}$ for some $\gamma>0$ (in the sense of Theorem III). 
Theorem IV is equivalent to:

(D) If $v_{0} \in H_{+} \cap\left(C_{u_{0}}-E_{u_{0}}\right)$, then any solution to (II-1) corresponding to $u_{0}$ and $v_{0}$ which exists on $[0, \infty)$ must grow at least as fast as $t^{2}$ (in the sense of Theorem IV). (See Figure II.)

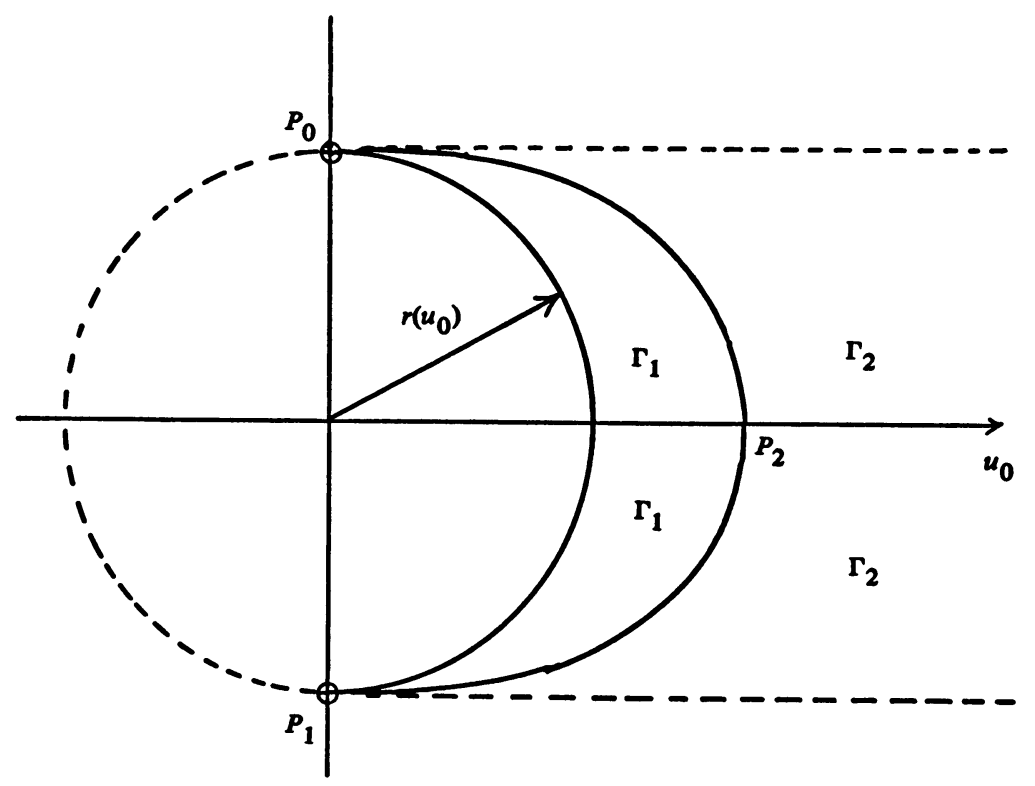

Figure II. If $v_{0} \in S_{4_{0}}$ or is on the "arc" $P_{0} P_{1}$ we have unbounded growth in finite time of $(u, P u)$. If $v_{0}$ belongs to region $\Gamma_{1}$ not including the "arc" $P_{0} P_{1}$ and excluding the "arc" $P_{0} P_{2} P_{1}$ we have at least exponential growth of $(u, P u)$. If $v$ belongs to region $\Gamma_{2}$, then we have at least quadratic polynomial growth of $(u, P u)$.

III. Examples. In this section we shall give some elementary applications of the preceding theorems. The list is not intended to be exhaustive. Moreover, due to considerations of length, we shall not give a completely rigorous verification of all of the hypotheses needed on $P, \mathscr{F}$ and $A(\cdot)$, but content ourselves with verification of only the more relevant conditions on these operators. For example, we shall not verify the regularity conditions on $\mathscr{F}$ but restrict our calculations to the formal verification of $(*)$ and (**). In most cases the Hilbert space norm on $D$ for which these regularity properties hold will be of the form

$$
\|x\|_{D}=\left[\|x\|^{2}+\lambda_{1}(P x, P x)+\lambda_{2}(A x, A x)\right]^{1 / 2} \text { for some } \lambda_{1}, \lambda_{2}>0 .
$$

The regularity properties of $\mathscr{F}$ themselves will then follow from the appropriate Sobolev inequality (assuming sufficient regularity in the solution space). 
Example I. Let $B \subset R^{n}$ be a bounded region with the boundary of $B, \partial B$, smooth enough to admit of applications of the divergence theorem. Let, for each $(\mathrm{x}, t) \in B \times(0, \infty), A(\mathbf{x}, t)=\left[a_{i j}(\mathrm{x}, t)\right]$ be an $n \times n$ symmetric matrix with continuously differentiable real valued entries such that

$$
\begin{aligned}
& \sum_{i, j=1}^{n} a_{i j}(\mathbf{x}, t) \xi_{i} \xi_{j} \geq 0 \text { for all } \xi=\left(\xi_{1}, \ldots, \xi_{n}\right) \in R^{n}, \\
& \sum_{i, j=1}^{n}\left(\frac{\partial}{\partial t} a_{i j}(\mathbf{x}, t)\right) \xi_{i} \xi_{j} \leq 0 \text { for all } \xi \in R^{n}
\end{aligned}
$$

Let the boundary of $B, \partial B$, be written as $\partial B=\Gamma_{1} \cup \Gamma_{2}$ where $\Gamma_{1}$ and $\Gamma_{2}$ are disjoint, "smooth" submanifolds of $B$. Suppose that $A(x, t)$ is independent of $t$ on $\Gamma_{2}$. We consider the problem

$$
\frac{\partial^{2} u}{\partial t^{2}}=\sum_{i, j=1}^{n} \frac{\partial}{\partial x_{i}}\left(a_{i j}(\mathbf{x}, t) \frac{\partial u}{\partial x_{i}}\right)+\mathscr{F}(u), \quad \mathscr{F}(0) \equiv 0,
$$

in $\Omega \times[0, \infty), u(x, t)=0$ on $\Gamma_{1} \times[0, \infty), \sum_{i, j=1} a_{i j}(x) \nu_{j} \partial u / \partial x_{i}=0$ on $\Gamma_{2} \times[0, \infty)$, where $\nu=\left(\nu_{1}, \ldots, \nu_{n}\right)$ is the outward directed normal (on $\left.\Gamma_{2}\right)$ to the boundary of $B$ and where $u(\mathbf{x}, 0)=u_{0}(\mathbf{x}),(\partial u / \partial t)(\mathbf{x}, 0)=v_{0}(\mathbf{x})$ are prescribed initially. Suppose that $\mathscr{F}(u)(\mathbf{x}, t)=\mathscr{F}(u(\mathbf{x}, t))$. Let $(f, g)=\int_{B} f \cdot g d \mathbf{x}$ denote the scalar product of the Hilbert space $H=\mathfrak{L}^{2}(B)$. We let $D=\left\{f \in H \mid f \in C^{2}(\bar{B})\right.$, $f=0$ on $\Gamma_{1}, \sum_{i, j=1} a_{i j}(\mathbf{x}) u_{x_{i}} \nu_{j}=0$ on $\left.\Gamma_{2}\right\}$ and, for $f \in D$

$$
[A(t) f](\mathbf{x})=-\sum_{i, j=1}^{n} \frac{\partial}{\partial x_{i}}\left(a_{i j}(\mathbf{x}, t) \frac{\partial}{\partial x_{j}}\right) f(\mathbf{x}) .
$$

One easily verifies that $A(\cdot)$ satisfies all the conditions on $A(\cdot)$ of Theorem I including condition (i) on $Q_{A}$. (In fact,

$$
Q_{A}(v, v)=\int_{B} \Sigma\left(\partial a_{i j} / \partial t\right)\left(\partial v / \partial x_{i}\right)\left(\partial v / \partial x_{j}\right) d \mathbf{x}
$$

for any smooth function $v(\mathbf{x}, t)$ such that for each $t, v(\cdot, t) \in D$.)

Since $P=I$, we take $D_{P}=H$.

The most interesting applications occur when either $\Gamma_{2}=\varnothing$ or $A(x, t)$ is independent of $t$.

We find from the definition of $\mathcal{G}$ that, for $f \in D$,

$$
\mathcal{G}(f)=\int_{B} \int_{0}^{1} \mathscr{F}(\rho f(\mathbf{x})) f(\mathbf{x}) d \rho d \mathbf{x}=\int_{B}\left(\int_{0}^{f(\mathbf{x})} \mathscr{F}(z) d z\right) d \mathbf{x}
$$

so that (*) holds for arbitrary $f \in D$ if and only if

$$
\int_{B}\left(\int_{0}^{f(x)}\left[\frac{d}{d z}(z \mathscr{F}(z))-(4 \alpha+2) \mathscr{F}(z)\right] d z\right) d \mathbf{x} \geq 0 .
$$


Thus, (*) holds if $z_{\mathscr{F}^{\prime}}(z)-(4 \alpha+1) \mathscr{F}(z) \geq 0$ for almost all $z>0$ and $z \mathscr{F}^{\prime}(z)$ $-(4 \alpha+1) \mathscr{F}(z) \leq 0$ for almost all $z<0$, or, equivalently,

$$
\mathscr{F}(z)=|z|^{4 \alpha+1} \varphi(z)
$$

where $\varphi: R^{1} \rightarrow R^{1}$ is monotone increasing (nondecreasing). Moreover, if we let $S_{+}=\left\{\mathbf{x} \in B \mid u_{0}(\mathbf{x})>0\right\}, S_{-}=\left\{\mathbf{x} \in B \mid u_{0}(\mathbf{x})<0\right\}$, we see that $\left(\mathrm{d}_{0}\right)$ holds if and only if

$$
\begin{aligned}
\mathcal{G}\left(u_{0}\right) & =\int_{S_{+}}\left(\int_{0}^{u_{0}(\mathbf{x})} \mathscr{F}(z) d z\right) d \mathbf{x}-\int_{S_{-}}\left(\int_{u_{0}(\mathbf{x})}^{0} \mathscr{F}(z) d z\right) d \mathbf{x} \\
& >\frac{1}{2}\left(u_{0}, A(0) u_{0}\right) .
\end{aligned}
$$

Thus, (III-2) can fail, for all $u_{0} \in D$, only when $\varphi \leq 0$ if $z \geq 0$ and $\varphi \geq 0$ if $z \leq 0$ (almost everywhere), i.e., only when $\varphi=0$ a.e. Therefore we have, for some $T<\infty$, if (III-2) holds and $v_{0} \in S_{u_{0},}, \lim _{t \rightarrow T^{-}} \int_{B} u^{2}(\mathbf{x}, t) d \mathbf{x}=+\infty$.

Remark. It is interesting to compare $\left(*^{\prime}\right)$ with the nonlinearities used by Jörgens [4] and Keller [5]. Although most of the conditions on $\mathfrak{G} \equiv \int_{0}^{z} \mathscr{F}(\eta) d \eta$ are different in all three cases, there is one condition that was to be satisfied by them all. Namely, the associated "potential" $\tilde{\mathcal{G}}$ satisfies, for some $z_{0}, \tilde{\mathcal{G}}(z)>0$ for all $z \geq z_{0}$ and $\int_{z_{0}}^{\infty} \tilde{g}^{-1 / 2}(z) d z<\infty$. That is, $\tilde{\mathcal{G}}^{-1 / 2}$ is integrable near infinity.

Example II. $A$ "compact" nonlinearity. In the preceding example, the nonlinearity had the form $\mathscr{F}(g)(\mathbf{x})=f(g(\mathbf{x})) g(\mathbf{x})$ where $f(s)=O\left(s^{8}\right)$ as $|s| \rightarrow+\infty$ for some $\delta>0$. In this example we take as the nonlinearity in (III-1)

$$
\mathscr{F}(g)(\mathbf{x})=g(\mathbf{x}) \int_{B} K(\mathbf{x}, \mathbf{y}) g^{2}(\mathbf{y}) d \mathbf{y}
$$

where $K$ is a square integrable, real valued function such that $K(\mathbf{x}, \mathbf{y})=K(\mathbf{y}, \mathbf{x})$. One easily finds that the Fréchet derivative of $\mathscr{F}$ at $g \in H$ is

$$
\mathscr{F}_{\mathrm{g}} \cdot h(\mathbf{x})=h(\mathbf{x}) \int_{B} K(\mathbf{x}, \mathbf{y}) g^{2}(\mathbf{y}) d \mathbf{y}+2 g(\mathbf{x}) \int_{B} K(\mathbf{x}, \mathbf{y}) g(\mathbf{y}) h(\mathbf{y}) d \mathbf{y}
$$

and that (F-I) and (F-II) hold since $\mathscr{F}_{g}$ is symmetric and bounded as a routine calculation shows. Also,

$$
\mathcal{G}(g)=\frac{1}{4} \int_{B} \int_{B} K(\mathbf{x}, \mathbf{y}) g^{2}(\mathbf{y}) g^{2}(\mathbf{x}) d \mathbf{x} d \mathbf{y}
$$

and $(4 \alpha+2) \mathcal{G}(g)=(\mathscr{F}(g), g)$ if $\alpha=\frac{1}{2}$. The condition $\left(\mathrm{d}_{0}\right)$ reads

$$
\int_{B} \int_{B} u_{0}^{2}(\mathbf{x}) u_{0}^{2}(\mathbf{y}) K(\mathbf{x}, \mathbf{y}) d \mathbf{y} d \mathbf{x}>2 \int_{B} \sum_{i, j}^{n} a_{i j}(\mathbf{x}, 0) u_{0, j} u_{0, j} d \mathbf{x} .
$$

Therefore, if $K$ is positive on a subset of $B$ of positive ( $n^{2}$ dimensional) Lebesgue measure, we can choose $u_{0} \in C_{0}^{2}(B)$ such that this last inequality is fulfilled. (This example can easily be generalized to more general integral operators.) 
Thus even a "smoothing out" of the nonlinearity makes no difference and instability can occur even in the above case.

Example III. Here we consider a very special case of Example I. Consider the problem

$$
\begin{aligned}
\partial^{2} u / \partial t^{2} & =\partial^{2} u / \partial x^{2}+\varepsilon \mathcal{F}(u), \quad(x, t) \in(0, a) \times[0, \infty), \\
u(x, 0) & =u_{0}(x), u_{t}(x, 0)=v_{0}(x), \quad x \in[0, a], \\
u(0, t) & =u(a, t)=0, \quad t \in[0, \infty) .
\end{aligned}
$$

We wish to examine the question of instability relative to the parameters $\varepsilon$ and a. If $\mathscr{F}(u)=u$, it is well known that all solutions to the above problem satisfy, for some $K>0$, if $\varepsilon \leq \pi^{2} / a^{2}$,

$$
\left\|u_{e}(t)\right\|_{a} \leq K\left(\left\|u_{0}\right\|_{a}+\left\|v_{0}\right\|_{a}\right) \quad(t \in[0, \infty))
$$

where $\|f\|_{a}=\left(\int_{0}^{a}|f|^{2} d x\right)^{1 / 2}$. Otherwise solutions may have at most exponential growth in time. We note that even here as $a \rightarrow+\infty$ the size of the set of $\varepsilon$ 's for which we have stability decreases although for any $\varepsilon<0$ and any $a>0$ we have the above stability inequality. However, if $\mathscr{F}(u)=u^{2}$ say, then for any $\varepsilon \neq 0$, there exist choices of $u_{0}$ and $v_{0}$ such that the corresponding solutions are unstable in finite time. (Here $S(f)=(1 / 3) \int_{0}^{a} f^{3}(x) d x, \alpha=\frac{1}{4}$.) To see this, let $f$ $\in C^{1}[0, a], f(x)>0$ if $x \in(0, a)$ and $f(0)=f(a)=0$. Let $u_{0}(x)=r f(x)$. Then $\left(\mathrm{d}_{0}\right)$ holds for $u_{0}$ provided

$$
\operatorname{\varepsilon r} \int_{0}^{a}(f(x))^{3} d x>\frac{3}{2} \int_{0}^{a}\left(f^{\prime}(x)\right)^{2} d x .
$$

Now for any given $\varepsilon$ simply choose $|r|$ large enough so that $\varepsilon r>0$ and such that the above inequality holds. In particular this says that for each $f$ in $\{f$ $\in C^{1}[0, a] \mid f(0)=f(a)=0, f>0$ on $\left.(0, a)\right\}$ and for each $\varepsilon \neq 0$ there is some $T>0$ and a number $r>0$ such that some solutions to the above initial boundary value problem with $u_{0}(x)=r f(x)$ become (pointwise) unbounded in $[0, a] \times[0, T)$. We note that in general, given $f$, we will have to take $\left\|u_{0}\right\|_{a}$ $=|r|\|f\|_{a}$ large. Consider, however $f(x)=(2 / a)^{1 / 2} \sin (\pi x / a)$, which is the first (normalized) eigenfunction corresponding to eigenvalue $\pi^{2} / a_{2}$ for $f^{\prime \prime}+\lambda f=0$, $f(0)=f(a)=0$. Let $u_{0}=r f$. Let $\varepsilon>0$ be fixed. We would like to choose $r>0$ so large that

$$
r \varepsilon \int_{0}^{a}(f(x))^{3} d x>3 \pi^{2} / 2 a^{2}
$$

i.e. so large that $\varepsilon r>K / a^{3 / 2}$ where $K$ is a positive constant independent of $a$ and $\varepsilon$. However, for fixed $\left\|u_{0}\right\|_{a}=r$, as $a$ increases for fixed $\varepsilon$, eventually there will be solutions to the above problem having initial displacement $u_{0}$ which will be unstable. Thus, in this sense, "large domains are more unstable than small domains" (Sattinger [14]). 
Example IV. $A$ higher order equation. The following example generalizes readily to higher dimensional problems: let $B \subset R^{2}$ be a bounded domain in the plane. We consider the following problem:

$$
\begin{aligned}
(2 \rho h / d) \partial^{2} w / \partial t^{2}= & -\left(\partial^{2} / \partial x^{2}+\partial^{2} / \partial y^{2}\right)^{2} w+\mathscr{F}(w) \text { in } B \times[0, \infty), \\
w(x, y, t)= & \frac{\partial}{\partial \nu} w(x, y, t)=0 \text { on } \partial B \times[0, \infty), \\
& w(x, y, 0), \frac{\partial w}{\partial t}(x, y, 0) \text { prescribed for }(x, y) \in B .
\end{aligned}
$$

This is the equation of motion of a clamped plate (see Love [13]) where $\mathscr{F}$ is a given loading function acting (vertically) on the plate. Here $\rho=\rho(x, y)$ is the density of the plate at $(x, y)$ while $d$ is the flexural rigidity and $h$ is the half thickness of the plate. We assume $\rho h / d>0$ at each point $(x, y) \in B$.

We take $H=\mathscr{L}^{2}(B)$ with the usual scalar product, and $A=\Delta^{2}$ where $D=D_{A}=\left\{f \in C^{4}(\bar{B}) \mid f=\partial f / \partial \nu=0\right.$ on $\left.\partial B\right\}$. We see that $(f, A f) \geq 0$ for all $f \in D$. If we let $\mathscr{F}(w)=\varepsilon w^{2}, f \in D$, be any function such that

$$
\varepsilon \int_{B} f^{3}(x, y) d x d y \geq \frac{3}{2} \int_{B}\left(f_{x x}+f_{y y}\right)^{2} d x d y,
$$

$w(x, y, 0)=f(x, y)$ and $w_{t}(x, y, 0)$ satisfy the conditions of Theorem $\mathbf{I}$, then the corresponding solution will become unbounded in finite time in the sense that $\lim _{t \rightarrow T^{-}} \int_{B} \rho h d^{-1} w^{2}(x, y, t) d x d y=+\infty$. (Note that in the linear problem we have stability. This follows from energy considerations.)

Observe also that the characteristics of $w_{t t}=-\Delta^{2} w$ are planes parallel to the $x y$ plane so that we do not have a Huyghen's principle and that the "domain of dependence" is the whole "initial plane". This precludes employment of the type of arguments used in [7], [9] and [14] to demonstrate instability of solutions to certain nonlinear wave equations.

Example V. $A$ system. In this example we consider the effect of a nonlinear forcing term added to the classical equations of linear elasticity, viz:

$$
\rho(\mathbf{x}) \frac{\partial^{2} u_{i}}{\partial t^{2}}=\frac{\partial}{\partial x_{k}}\left(c_{i j k l}(\mathbf{x}) \frac{\partial u_{j}}{\partial x_{l}}\right)+\mathscr{F}_{i}\left(u_{1}, u_{2}, u_{3}\right), \quad i=1,2,3,
$$

in $B \times[0, \infty)$. (The summation convention is employed here.) In vector notation

$$
\rho(\mathbf{x}) \partial^{2} \mathbf{u} / \partial t^{2}=\left(\partial / \partial x_{k}\right)\left(C_{k l}(\mathbf{x}) \partial u / \partial x_{l}\right)+\overrightarrow{\mathscr{F}}(\mathbf{u})
$$

The matrices $C_{k l}=\left(c_{i k j l}\right)$ are assumed to satisfy $c_{i k j l} \eta_{j l} \eta_{i k} \geq 0$ for all $\eta=\left[\eta_{i j}\right]_{3 \times 3}$ with real entries and all $\mathrm{x} \in \bar{B}$. (We presuppose the symmetry $C_{l k}=C_{k l}^{T}$ or $c_{i k j l}=c_{j l i k .}$.) We assume that the solution $\mathbf{u}=\operatorname{col}\left(u_{1}, u_{2}, u_{3}\right)$ satisfies some standard homogeneous boundary conditions. (For example, in the notation used in Example $\mathrm{I}, \mathrm{u}(\mathrm{x}, 0)=0$ on $\Gamma_{1} \times[0, \infty)$ while $\nu_{k} c_{i k j l} u_{j, l}=0$ for $i=1,2,3$ on 
$\Gamma_{2} \times[0, \infty)$.) This condition together with the appropriate smoothness conditions on u serves as the defining condition for the domain of the operator

$$
A \mathbf{u}=\left[C_{k l} \mathbf{u}, l\right]_{k} .
$$

(The Hilbert space $H=\mathfrak{L}^{2}(B) \oplus \mathfrak{L}^{2}(B) \oplus \mathfrak{L}^{2}(B)$ is of course equipped with the scalar product $(\mathbf{u}, \mathbf{v})=\int_{B} \sum u_{i} v_{i} d \mathbf{x}$.)

We take as our nonlinearity a vector function

$$
\overrightarrow{\mathscr{F}}(\mathbf{u})(\mathbf{x}, t)=\overrightarrow{\mathscr{F}}(\mathbf{u}(\mathbf{x}, t)), \quad \overrightarrow{\mathscr{F}}\left(u_{1}, u_{2}, u_{3}\right)=\operatorname{col}\left(\mathscr{F}_{1}, \mathscr{F}_{2}, \mathscr{F}_{3}\right) .
$$

Let $\left(\partial \mathscr{F}_{i} / \partial u_{j}\right)=\left(\partial \mathscr{F}_{j} / \partial u_{i}\right), i, j=1,2,3$, so that $\overrightarrow{\mathscr{F}}=\nabla \mathcal{F C}$ where $\mathcal{H}=\mathcal{H C}\left(u_{1}, u_{2}, u_{3}\right)$ is a scalar function (potential). From the definition of $\mathcal{G}$ in (F-II) (assuming $\mathcal{F}(0,0,0)=0)$ and for $f$ in the appropriate domain,

$$
\begin{aligned}
\mathcal{G}(\mathbf{f}) & =\mathcal{G}\left(f_{1}, f_{2}, f_{3}\right)=\int_{0}^{1}\left(\int_{B} \mathscr{F}_{i}(\boldsymbol{\rho f}(\mathbf{x})) f_{i}(\mathbf{x}) d \mathbf{x}\right) d \rho \\
& =\int_{B}\left(\int_{0}^{1} \mathcal{F}_{,_{i}}(\boldsymbol{\rho} \mathbf{f}) f_{i} d \rho\right) d \mathbf{x}=\int_{B} \mathcal{F}(\mathbf{f}(\mathbf{x})) d \mathbf{x} .
\end{aligned}
$$

It follows that (*) will hold for any solution to (III-4) if

$$
(4 \alpha+2) \mathcal{H C}\left(\xi_{1}, \xi_{2}, \xi_{3}\right) \leq \sum_{i=1}^{3} \xi_{i} \frac{\partial \mathcal{Y}}{\partial \xi_{i}}\left(\xi_{1}, \xi_{2}, \xi_{3}\right)
$$

while $\left(d_{0}\right)$ holds for the initial displacement $u(x, 0)$ if

$$
\int_{B} \mathcal{T}(\mathbf{u}(\mathbf{x}, 0)) d \mathbf{x}>\frac{1}{2} \int_{B} c_{i k j l}(\mathbf{x}) \frac{\partial u_{i}(\mathbf{x}, 0)}{\partial x_{k}} \cdot \frac{\partial u_{j}(\mathbf{x}, 0)}{\partial x_{l}} d \mathbf{x} .
$$

(The summation convention is employed here.) Unfortunately, it is not possible to give as simple a characterization of all the scalar functions $\mathcal{H}$ satisfying $\left(*^{\prime \prime}\right)$ as in the one dimensional case $\left(*^{\prime}\right)$. However, if $\mathcal{I C}$ is homogeneous of degree $4 \alpha+2$, then (*") holds with equality (Euler's identity).

The corresponding initial velocity vectors $\mathbf{u}_{t}(\mathbf{x}, 0)$ can be chosen as indicated by Theorem II. Thus, for such choices, there is $T, 0<T<\infty$, such that

$$
\lim _{t \rightarrow T^{-}} \int_{B} \rho u_{i}(\mathbf{x}, t) u_{i}(\mathbf{x}, t) d \mathbf{x}=+\infty
$$

but one cannot say which of the three integrals in (III-6) is the unbounded one.

Example VI. If one considers the extensional vibration of a rod of uniform cross section fixed at one end and free at the other, then the governing equation has the form $\rho w_{t t}=E w_{x x}$ where $\rho$ is the (uniform) density of the material, $E$ is Young's modulus, and $w$ is the displacement. If, however, the inertia of the lateral motion is taken into account, the governing equation (Love [13, p. 428]) becomes

$$
\rho\left(\partial^{2} / \partial t^{2}\right)\left(w-\sigma^{2} K^{2} \partial^{2} w / \partial x^{2}\right)=E \partial^{2} w / \partial x^{2}
$$

where $\sigma$ is Poisson's ratio and $K$ is the radius of gyration of a cross-section about a central line. At the fixed end $w=0$; at the free end $\partial w / \partial x=0$. 
Therefore, we are led to consider nonlinear problems of the form

$$
\frac{\partial^{2}}{\partial t^{2}}\left[\rho(\mathbf{x})-\frac{\partial}{\partial x_{i}}\left(a_{i j}(\mathbf{x}) \frac{\partial}{\partial x_{j}}\right)\right] u=\frac{\partial}{\partial x_{i}}\left(b_{i j}(\mathbf{x}) \frac{\partial}{\partial x_{j}}\right) u+\mathscr{F}(u)
$$

where $\left[a_{i j}(\mathbf{x})\right]$ and $\left[b_{i j}(\mathbf{x})\right]$ are positive definite and semidefinite matrices respectively at each $x$ in a suitable subset of $R^{n}$. (Here $\rho \geq 0$. If $\rho>0$ then $\left[a_{i j}\right]$ need only be positive semidefinite.)

If the nonlinearity is taken as in Examples I or II and if $u_{0}(x) \equiv u(x, 0)$ satisfies $\left(d_{0}\right)$, then one can apply the conclusions of Theorem I to the appropriate initial-boundary value problem for (III-7) to deduce the nonexistence of global solutions if $v_{0}$ is appropriately chosen.

However, one cannot apply the arguments of [9] and [14] to (III-7) even if one had available a "finite speed of propagation principle" because if $\rho(x)$ is not constant then one cannot free the equation of the space variable in order to get a first (and second) integral of an associated ordinary differential equation whose solutions blow up in finite time and solve the partial differential equation.

However, one may use our results in conjunction with the (known) finite speed of propagation principle for either the initial value problem or an initialboundary value problem for

$$
\rho(x) \partial^{2} u / \partial t^{2}=\partial^{2} u / \partial x^{2}+u^{2}(x, t) \quad\left(\rho>0 \text { on } R^{1}\right)
$$

to conclude that if $u_{0}$ and $v_{0}$ have compact support and if they satisfy $\left(\mathrm{d}_{1}\right)$ of Theorem I, then the corresponding solution to (III-8) becomes pointwise unbounded in finite time.

Finally, we remark that it is possible to treat the nonlinear version of the following equation of motion of a naturally bent rod, namely

$$
\left(I-\frac{\partial^{2}}{\partial \theta^{2}}\right) \frac{\partial^{2} w}{\partial t^{2}}=c^{2}\left(\frac{\partial^{6} w}{\partial \theta^{6}}+2 \frac{\partial^{4} w}{\partial \theta^{4}}+\frac{\partial^{2} w}{\partial \theta^{2}}\right) \quad\left(\theta \in\left[0, \theta_{0}\right], 0<\theta_{0}<2 \pi\right)
$$

(see [13]).

Note, moreover, that if one begins with a problem of the form (II-1) which is an abstraction of an initial-boundary value problem and if the region in space under consideration is bounded, then $L^{2}$ growth of the solution (assumed continuously differentiable) will imply pointwise growth irrespective of whether the $L^{2}$ growth to infinity takes place in finite or infinite time. If $P$ is a bounded operator, growth of $(u, P u)$ implies growth of $(u, u)$ clearly. If $P$ is not bounded, say $P=-d^{2} / d x^{2}$, then we get pointwise growth of $\partial u / \partial x$.

IV. The damped equation. In the notation of $\S I$, we suppose that $P=I$, $A \geq 0$ and, for simplicity, that $A(t)=A$ independent of $t$. Our problem is

$$
u_{t t}+a u_{t}=-A u+\mathscr{F}(u), \quad u(0)=u_{0}, \quad u_{t}(0)=v_{0},
$$


where $a>0$ is constant. (We assume the same regularity as for solutions to (II-1).)

Theorem VI. Suppose $\mathcal{F}$ and $A$ satisfy the hypotheses of Theorem I. Let $u:[0, T) \rightarrow H$ be a solution to (IV-1) in the sense of a definition analogous to that used in Theorem I for solutions to (II-1). Suppose that

$$
\mathcal{G}\left(u_{0}\right)>\frac{1}{2}\left[\left(u_{0}, A u_{0}\right)+(a / 2 \alpha)^{2}\left(u_{0}, u_{0}\right)\right] .
$$

Let

$$
\cos \theta=\left(u_{0}, v_{0}\right) /\left\|u_{0}\right\|\left\|v_{0}\right\|, \quad r\left(u_{0}\right)=\sqrt{ } 2\left[\mathcal{G}\left(u_{0}\right)-\frac{1}{2}\left(u_{0}, A u_{0}\right)\right]^{1 / 2} .
$$

Then, for any $v_{0} \in D$ such that $\left\|v_{0}\right\| \leq r\left(u_{0}\right)$ and $\left\|v_{0}\right\| \cos \theta>a\left\|u_{0}\right\| / 2 \alpha$, the interval of existence of this solution is finite and

$$
\lim _{t \rightarrow T^{-}}\|u(t)\|=+\infty
$$

where

$$
T \leq a^{-1} \ln \left\{2 \alpha\left\|v_{0}\right\| \cos \theta /\left(2 \alpha\left\|v_{0}\right\| \cos \theta-a\left\|u_{0}\right\|\right)\right\}
$$

Proof. Let $F(t)=(u, u)$. Then $F^{\prime}(t)=2\left(u, u_{t}\right)$ and $F^{\prime \prime}(t)=2\left(u, u_{t t}\right)+2\left(u_{t}, u_{t}\right)$ so that

$$
F F^{\prime \prime}-(\alpha+1)\left(F^{\prime}\right)^{2} \geq 4(\alpha+1) S^{2}+2 F\left\{\left(u, u_{t}\right)-(2 \alpha+1)\left(u_{t}, u_{t}\right)\right\}
$$

Let

$$
\begin{aligned}
H(t) & =\left(u, u_{t}\right)-(2 \alpha+1)\left(u_{t}, u_{t}\right) \\
& =-(u, A u)-a\left(u_{t}, u\right)+(u, \mathscr{F}(u))-(2 \alpha+1)\left(u_{t}, u_{t}\right)
\end{aligned}
$$

Then

$$
\begin{aligned}
H^{\prime}(t)= & \left(u, u_{t t}\right)-(4 \alpha+1)\left(u_{t}, u_{t t}\right) \\
= & -a\left(u, u_{t t}\right)+4 \alpha\left(u_{t}, A u\right)+a(4 \alpha+1)\left(u_{t}, u_{t}\right) \\
& +d(u, \mathcal{F}(u)) / d t-(4 \alpha+2)\left(u_{t}, \mathscr{F}(u)\right) .
\end{aligned}
$$

Thus, integrating back, using (*), (**) and $\left(D_{0}\right)$,

$$
\begin{aligned}
H(t) & \geq H(0)-a \int_{0}^{t} \frac{d}{d \eta}\left(u, u_{\eta}\right) d \eta+(4 \alpha+2) a \int_{0}^{t}\left(u_{\eta}, u_{\eta}\right) d \eta-2 \alpha\left(u_{0}, A u_{0}\right) \\
(\mathrm{IV}-4) \quad & \geq-a\left(u, u_{t}\right)+2(2 \alpha+1) \mathcal{S}\left(u_{0}\right)-(2 \alpha+1)\left[\left(u_{0}, A u_{0}\right)+\left(v_{0}, v_{0}\right)\right] \\
& \geq-a F^{\prime}(t) / 2 .
\end{aligned}
$$


Therefore $F F^{\prime \prime}-(\alpha+1)\left(F^{\prime}\right)^{2} \geq-a F F^{\prime}$ so that $\left(F^{-\alpha}\right)^{\prime \prime}+a\left(F^{-\alpha}\right)^{\prime} \leq 0$ or $\left[e^{a t}\left(F^{-\alpha}\right)^{\prime}\right]^{\prime}<0$. Integrating this latter inequality back twice, we find that

$$
F^{\alpha}(t) \geq F^{\alpha}(0)\left\{1-\left(1-e^{-a t}\right) \alpha F^{\prime}(0) / a F(0)\right\}^{-1} .
$$

Now the expression in braces on the right of (IV-5) will vanish at some finite $t_{0}>0$ provided $\alpha F^{\prime}(0) / a F(0)>1$, i.e. provided $\left(u_{0}, v_{0}\right)>(a / 2 \alpha)\left(u_{0}, u_{0}\right)$ which is the case by hypothesis. Therefore, $\lim _{t \rightarrow T^{-}}\|u(t)\|=+\infty$ where $T \leq t_{0}$ and

$$
\begin{aligned}
t_{0} & =a^{-1} \ln \left[\alpha F^{\prime}(0) /\left(\alpha F^{\prime}(0)-a F(0)\right)\right] \\
& =a^{-1} \ln \left[2 \alpha\left(u_{0}, v_{0}\right) /\left(2 \alpha\left(u_{0}, v_{0}\right)-a\left(u_{0}, u_{0}\right)\right)\right] .
\end{aligned}
$$

The result follows.

The theorem has the geometric interpretation shown in Figure III.

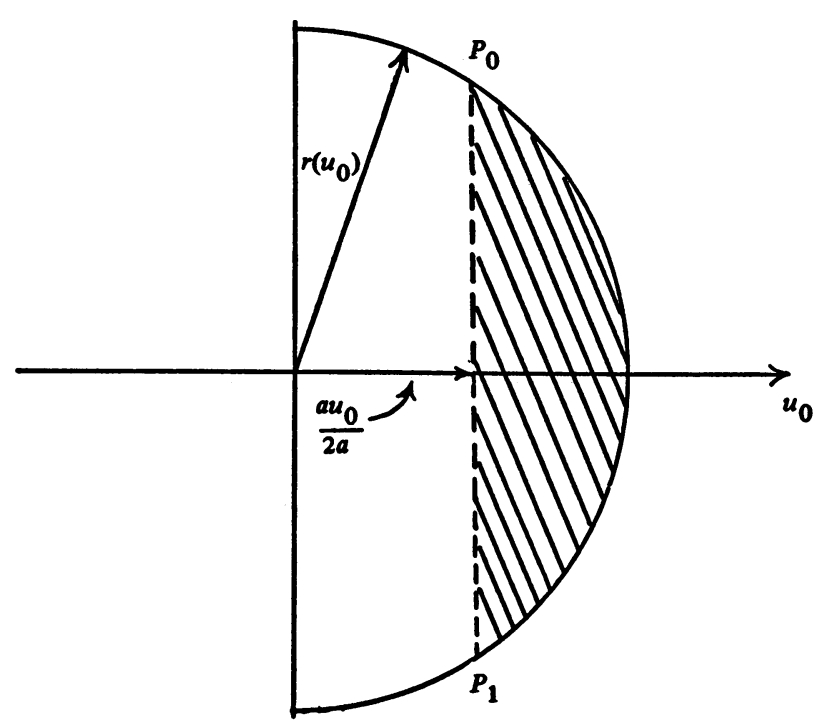

Figure III. If $v_{0}$ lies in the shaded region including the boundary of the sphere to the right of the hyperplane indicated by the segment $P_{0} P_{1}$, then $\lim _{t \rightarrow T}-\|u(t)\|=+\infty$.

Remark. Note that if, in addition, $\mathcal{F}$ satisfies the hypothesis of Corollary I-1, then there exist $u_{0}$ 's in $D$ such that $\left(D_{0}\right)$ holds and consequently there are $v_{0}$ 's such that $\left\|v_{0}\right\| \leq r\left(u_{0}\right)$ and $\left\|v_{0}\right\| \cos \theta>a\left\|u_{0}\right\| / 2 \alpha$.

Note also that, in contrast to [14], it was not necessary to use any special properties of $\boldsymbol{A}$ or of any associated ordinary differential equation.

V. Concluding remark. A similar analysis has been carried out for abstract equations of the form 


$$
P d u / d t=-A(t) u+\mathscr{F}(u)
$$

where $P>0$ and $A \geq 0$. (See [16].)

Acknowledgements. The author would like to thank Dr. William Faris of the Battelle Advanced Studies Center of Geneva for suggesting the problem to him and Professor L. E. Payne for some valuable suggestions which improved an earlier version of this work.

Note added later. Theorem VI can be improved somewhat by putting $F(t)$ $=(u, u)+\beta(t+\tau)^{2}$ as in Theorem I. We have

Theorem VII. Let $\mathscr{F}$ and $A$ satisfy the hypotheses of Theorem $\mathrm{I}$ and $u:[0, T) \rightarrow H$ solve (IV-1) in the sense of Theorem I. Suppose that $\left(D_{0}\right)$ of Theorem VI holds. Let $r\left(u_{0}\right)=\sqrt{ } 2\left[\mathcal{S}\left(u_{0}\right)-\frac{1}{2}\left(u_{0}, A u_{0}\right)\right]^{1 / 2}$. Then for any $v_{0} \in D$ such that $v_{0} \in S_{u_{0}} \cap \bar{S}_{a, u_{0}}$ where

$$
\bar{S}_{a, u_{0}} \equiv\left\{v_{0} \in D \mid\left\|a u_{0} / \alpha-v_{0}\right\| \leq r\left(u_{0}\right)\right\},
$$

there exists $T, 0<T<\infty$, such that $\lim _{t \rightarrow T^{-}}(u, u)=+\infty$.

Proof. With $F(t)=(u, u)+\beta(t+\tau)^{2}$, we find that

$$
F F^{\prime \prime}-(\alpha+1)\left(F^{\prime}\right)^{2} \geq 4(\alpha+1) S^{2}+2 F H(t)
$$

where $S^{2}$ is given for $P=I$ by the equation following (II-3) and

$$
H(t)=\left(u, u_{t t}\right)-(2 \alpha+1)\left(u_{t}, u_{t}\right)-(2 \alpha+1) \beta .
$$

By a calculation analogous to that leading to (IV-4) we see that

$$
H(t) \geq-a\left(u, u_{t}\right)+2(2 \alpha+1)\left[\mathcal{G}\left(u_{0}\right)-\frac{1}{2}\left(u_{0}, A u_{0}\right)-\frac{1}{2}\left(v_{0}, v_{0}\right)-\frac{1}{2} \beta\right] .
$$

Setting $\beta=2\left[G\left(u_{0}\right)-\frac{1}{2}\left(u_{0}, A u_{0}\right)\right]-\left(v_{0}, v_{0}\right)=r^{2}\left(u_{0}\right)-\left\|v_{0}\right\|^{2}>0$ and observing that $F^{\prime}(t)=2\left(u, u_{t}\right)+2 \beta(t+\tau) \geq 2\left(u_{t}, u\right)$, we find that $H(t) \geq-\frac{1}{2} a F^{\prime}(t)$ and hence

$$
F F^{\prime \prime}-(\alpha+1)\left(F^{\prime}\right)^{2} \geq-a F F^{\prime}
$$

Therefore (IV-5) holds for this choice of $F$. The conclusion of the theorem follows if we can show that $\alpha F^{\prime}(0) / a F(0)>1$. This is possible for some $\tau>0$ provided there exists $\tau$ such that

$$
P(\tau) \equiv \tau^{2}-2 \alpha \tau / a+\left[\left\|u_{0}\right\|^{2}-2 \alpha\left(u_{0}, v_{0}\right) / a\right] \beta^{-1}<0 .
$$

This is the case if

$$
0<\tau<\alpha / a+\left\{(\alpha / a)^{2}-\left[\left\|u_{0}\right\|^{2} / \beta-2 \alpha\left(u_{0}, v_{0}\right) / a \beta\right]\right\}^{1 / 2} .
$$

This is clearly possible provided $r\left(u_{0}\right)^{2} \geq\left\|a u_{0} / \alpha-v_{0}\right\|^{2}$, as we see from a computation using the definition of $\beta$ and the condition that the discriminant of $P(\tau)$ be nonnegative. 
Since $t_{0}=-a^{-1} \ln \left[1-a F(0) / \alpha F^{\prime}(0)\right]$ we see that $t_{0}$ will be as small as possible provided $\alpha F^{\prime}(0) / a F(0)$ is as large as possible.

We see that $S_{u_{0}} \cap \bar{S}_{a, u_{0}} \neq \varnothing$ if and only if $2 r\left(u_{0}\right)>(a / \alpha)\left\|u_{0}\right\|$. This latter inequality is simply a restatement of $\left(D_{0}\right)$. The geometrical interpretation of this statement is self evident.

It is of interest to remark that if we find a $u_{0}$ such that $r\left(u_{0}\right)>0$, then in any problem where the damping constant $a$ is less than $2 \alpha r\left(u_{0}\right) /\left\|u_{0}\right\|$, the theorem holds for $v_{0} \in S_{u_{0}} \cap \bar{S}_{a, u_{0}} \neq \varnothing$. Moreover, if the damping constant is smaller than $\alpha r\left(u_{0}\right) /\left\|u_{0}\right\|$ then there are $v_{0}$ 's in $S_{u_{0}} \cap \bar{S}_{a, u_{0}}$ such that $\left(u_{0}, v_{0}\right) \leq 0$. Then, even for such pairs of initial data, $u_{0}, v_{0}$, the solutions will not be global.

\section{REFERENCES}

1. N. Bazley and B. Zwahlen, A branch of positive solutions to nonlinear eigenvalue problems, Manuscripta Math. 2 (1970), 365-374. MR 42 \#3628.

2.-, Estimation of the bifurcation coefficient for nonlinear eigenvalue problems, Z. Angew. Math. Phys. 20 (1969), 281-288. MR 40 \#6314.

3. Stephen Berman, Abstract wave equations with finite velocity of propagation, Bull. Amer. Math. Soc. 77 (1971), 1011-1013.

4. A. Friedman, Partial differential equations, Holt, Rinehart and Winston, New York, 1969.

5. F. John, Continuous dependence on data for solutions of differential equations with a prescribed bound, Comm. Pure Appl. Math. 13 (1960), 551-585. MR 24 \# A317.

6. K. Jörgens, Das Anfangswertproblem im Grossen für eine Klasse Nichtlinearer Wellengleichung, Math. Z. 77 (1967), 295-308.

7._, Nonlinear wave equations, University of Colorado Report, Boulder, Col., 1970.

8. J. B. Keller, On solutions of $\Delta u=f(u)$, Comm. Pure Appl. Math. 10 (1957), 503-510. MR 19, 964.

9. - On solutions of nonlinear wave equations, Comm. Pure Appl. Math. 10 (1957), 523-530. MR 20 \#3371.

10. R. J. Knops, H. A. Levine and L. E. Payne, Nonexistence, instability and growth theorems for solutions of a class of abstract nonlinear equations with applications to nonlinear elastodynamics (to appear).

11. H. A. Levine, Logarithmic convexity and the Cauchy problem for some abstract second order differential inequalities, J. Differential Equations 8 (1970), 34-55. MR 41 \#3945.

12. H. A. Levine and L. E. Payne, A nonexistence theorem for the heat equation with a nonlinear boundary condition and for the porus medium equation, backward in time (to appear).

13. A. E. H. Love, A treatise on the mathematical theory of elasticity, Dover, New York, 1944. MR $6,79$.

14. D. H. Sattinger, Stability of nonlinear hyperbolic equations, Arch. Rational Mech. Anal. 28 (1967/68), 226-244. MR 37 \#567.

15. W. A. Strauss, The energy method in nonlinear partial differential equations, Math. Notes 47, Instituto de Matematica Pura e Aplicada, Rio de Janeiro, Brazil, 1969. MR 42 \#8051.

16. H. A. Levine, Some nonexistence and instability theorems for solutions of formally parabolic equations of the form $P u_{t}=-A u+F(u)$, Arch. Rational Mech. Anal. (in print).

The School of Mathematics, Institute of Technology, University of Minnesota, MinneAPOLIS MinNESOTA 55455

Current address: Department of Mathematics, University of Rhode Island, Kingston, Rhode Island 02881 